\title{
Resource Cube: Multi-Virtual Resource Management for Integrated Satellite-Terrestrial Industrial IoT Networks
}

\author{
Danyang Chen*, Chungang Yang*, Peng Gong ${ }^{\dagger}$, Lizhong Chang*, Junqi Shao*, Qiang $\mathrm{Ni}^{\ddagger}$, Alagan \\ Anpalagan $^{\S}$, Mohsen Guizani ${ }^{\uparrow}$
}

\begin{abstract}
Industrial Internet of Things (IIoT) has found wider research, and satellite-terrestrial network (STN) can provide large-scale seamless connections for IIoT. With virtualization, we design resource cube to describe the integration and state of multi-dimensional virtual resources. To achieve higher resource utilization and smarter connections, we design a matching considered preferences (MCPR) algorithm to match IIoT nodes with service sides. The matching design considers the resource cube (MCRC) algorithm based on MCPR algorithm to lower the total system delay. In addition, in order to simplify the analysis of resource management, we adopt a layered architecture and multiple $\mathrm{M} / \mathrm{M} / \mathbf{1}$ queuing models. We analyze the resource utilization and the total system delay for three different combinations of arrival rate and service rate of each resource cube. With MCRC algorithm, the utilization of resources is slightly reduced, while the total system delay is greatly reduced compared with MCPR algorithm.
\end{abstract}

Index Terms-Internet of Things, multi-virtual resource management, satellite-terrestrial network, virtualization

Copyright (c) 2015 IEEE. Personal use of this material is permitted. However, permission to use this material for any other purposes must be obtained from the IEEE by sending a request to pubspermissions@ieee.org.

* D. Chen, C. Yang, L. Chang, and J. Shao are with the State Key Laboratory on Integrated Services Networks, Xidian University, Xi'an, 710071 China (emails: Cdanyang_chen@163.com; chgyang2010@163.com; lzchang.66@foxmail.com; ruya0905@163.com); and C. Yang is also supported by National Mobile Communications Research Laboratory, Southeast University.

$\dagger \mathrm{P}$. Gong is with Beijing Institute of Technology, Beijing, 100081, China (email: penggong@bit.edu.cn).

$\ddagger \mathrm{Q}$. Ni is with School of Computing and Communications, Lancaster University, Lancaster, UK. (email: q.ni@lancaster.ac.uk).

8 A. Anpalagan is with the Department of Electrical and Computer Engineering, Ryerson University, Toronto, ON, Canada (email: alagan@ee.ryerson.ca)

I M. Guizani is with Electrical and Computer Engineering Department University of Idaho, Idaho, USA. (email: mguizani@ieee.org).

This work was supported in part by the National Science Foundation of China (61871454); by the open research fund of National Mobile Communications Research Laboratory, Southeast University (No. 2019D10); by the Fundamental Research Funds for the Central Universities (JB190119); by the National Science Foundation of China under Grants 61671062; by the CETC Key Laboratory of Data Link Technology (CLDL-20182308). Prof. P. Gong's work was supported in part by the National Key Research and Development Program of China under Grant 2018YFC0823003 and the National Natural Science Foundation of China under Grant 61671062

\section{INTRODUCTION}

With the development of the next generation networks and the Industry 4.0, there will be multiple traffic types of Internet applications under different network scenarios, leading to different requirements and different representative applications. For example, Machine Type Communication represented by the IoT, whose purpose is to realize the connectivity of all things around us. Enhanced mobile broadband pursues the ultimate communication experience between people, which requires large traffic, wide frequency band and high frequency utilization [1]. Self-driving cars are a representative application of Ultra Reliable Low Latency Communications, which requires low latency and reliable connectivity [2]. Among these new and diverse network scenarios and applications, the Industrial IoT (IIoT) plays an important role with the development of Industry 4.0.

Predicted by Cisco, there will be 50 billion devices connected to the Internet by 2020 [3]. Furthermore, the continuous development of the IIoT puts forward higher requirements for network service capability. First of all, with the rise of emerging applications, the IIoT has proposed a broader need for network coverage, even beyond the terrestrial network coverage. Meanwhile, a large number of IIoT applications increase the demand for network resources, and the terrestrial network is difficult to provide network resources that meet all IIoT requirements in some cases. As a result, single terrestrial networks can hardly meet requirements of multi-emerging IIoT applications.

Since the satellite networks are characterized by large coverage and high delay, and the terrestrial networks are characterized by small coverage and low delay, jointly considering the terrestrial networks and the satellite networks, i.e., the integrated satellite-terrestrial network (STN), is the future development trend of network. Besides, different satellites have different features. For instance, low earth orbit (LEO) satellites have lower latency with lower pass over time [4]. Medium earth orbit (MEO) satellites and geostationary orbit (GEO) satellites are characterized by broader coverage but longer delay [5]. Therefore, the combination of multi-layer satellites and the terrestrial networks can provide different services 
according to different needs. And it can provide more flexible and better services for IIoT applications. Because the satellite networks and the terrestrial networks have different characteristics, they can complement each other. Meanwhile, the integration of resources of the satellite networks and the terrestrial networks can bring more benefits and achieve more efficient and rational resource utilization. More importantly, the STN can accomplish tasks that are difficult to accomplish in a single network.

In the meantime, using the STN to provide services for IIoT applications has become a tendency in industry and academia [6]-[11]. As mentioned in [6], an integrated satellite-terrestrial network can support IoT with virtualization. In [7], the authors demonstrate the advantage of using the LEO satellites to provide services for IoT applications, and point out that the LEO satellites can play an irreplaceable role in the development of the IoT. Using the STN to support applications of the Internet of remote things is presented in [8] with important issues about applications using heterogeneous networks and the promising enormous advantages. The development of the STN can bring much more benefits for IIoT applications. Besides, different characteristics of the terrestrial and the satellite networks mean that they cannot simply be mixed together. In order to simplify the process, the hierarchical architecture [12] [13] is adopted to provide services for broader coverage of IIoT applications.

Meanwhile, in order to manage multi-dimensional resources in the STN, we adopt the virtualization technology. There have also been some studies on the use of virtualization in the STN [14]-[26]. Among those studies, authors in [14]-[16] studies the architecture of the STN. Authors in [14] consider a satellite-terrestrial architecture with software defined features, and analyze key performances between its resource management schemes. And authors in [15] studies using software defined network $(\mathrm{SDN})$ in the design of architecture in multi-layered STN. Authors in [16] propose an integrated architecture based on SDN for managing cache resources. In addition to studies of architecture, there are some studies about resources management in the STN. Authors in [18] analyze three dynamic resource request strategies in the satellite networks based on queue volume and arrival rate. A cloudbased integrated satellite-terrestrial network is proposed in [20], considering a scenario that the terrestrial and the satellite networks share the same frequency band. Authors in [22] conclude the work related to the space information network using SDN/NFV, and propose a three-tier integrated space satellite framework based on the previous work. They propose two heuristic algorithms to provide fine-grained QoS assurance for multiple users. And there also researches about the satellite gateway placement. Authors in [23] studies the optimal configuration of satellite gateway in the STN. And in [24], authors further studies the optimal configuration of STN network satellite gateway under the condition of latency minimization with reliability guarantee. Hence, using virtualization to mix the terrestrial and the satellite networks and the joint management of multi-dimensional resources are important trends. Meanwhile, in order to allocate resources flexibly and improve resource utilization, we consider using the technology of virtualization to implement the orchestration of computation, communication and storage resources in STN. Those existing researches is mainly about the management of one or two kinds of resources, multidimensional resources are not considered.

In order to achieve efficient management of multidimensional resources based on IIoT applications in the STN, we have designed a utility function that maximizes resource utilization within pre-defined time delay. Since there are heterogeneous networks in STN, we choose matching game to determine the network node that provides services for IIoT applications. As the number of IIoT nodes increases, the network scale also becomes very large. Meanwhile, efficient resource allocation schemes usually require global information from the network which is difficult to obtain as the network scale increases. While Markov approximation can solve the problem that the optimization problem is difficult to deal with due to the complexity of network scale and the lack of global information. Hence we adopt the mean of matching with Markov approximation and Markov chain [27]-[31]. Among the existing work, authors in [28] summarize some game theories used in resource management of IoT systems. In addition, authors in [29] use Markov chain as an auxiliary with game theory to analyze resource management in the satellite networks.

The contributions of this paper are as follows:

- We design resource cubes to depict the combination of virtual communication, computation and storage resources and implement a simple description of resource state transfer based on the concept of resource cubes.

- According to the quantity of resource cubes, we set virtual resource controllers to adjust service sides matched by part of IIoT nodes intelligently. By adjusting the matching results of these IIoT nodes, unnecessary queuing delay can be avoided.

- For getting the steady-state probability involved in using Markov approximation, we use a new method based on Markov chain.

The remainder of this paper is organized as follows. We describe the system architecture in section II. Section III describes resource cubes we designed. In Section IV, we describe matching games between IIoT nodes and network service sides, and then in Section V use Markov chain and Markov approximation to deal with the resource utilization maximization problem. And Section VI and VII give simulation results, analysis and conclusions of the whole paper respectively. 


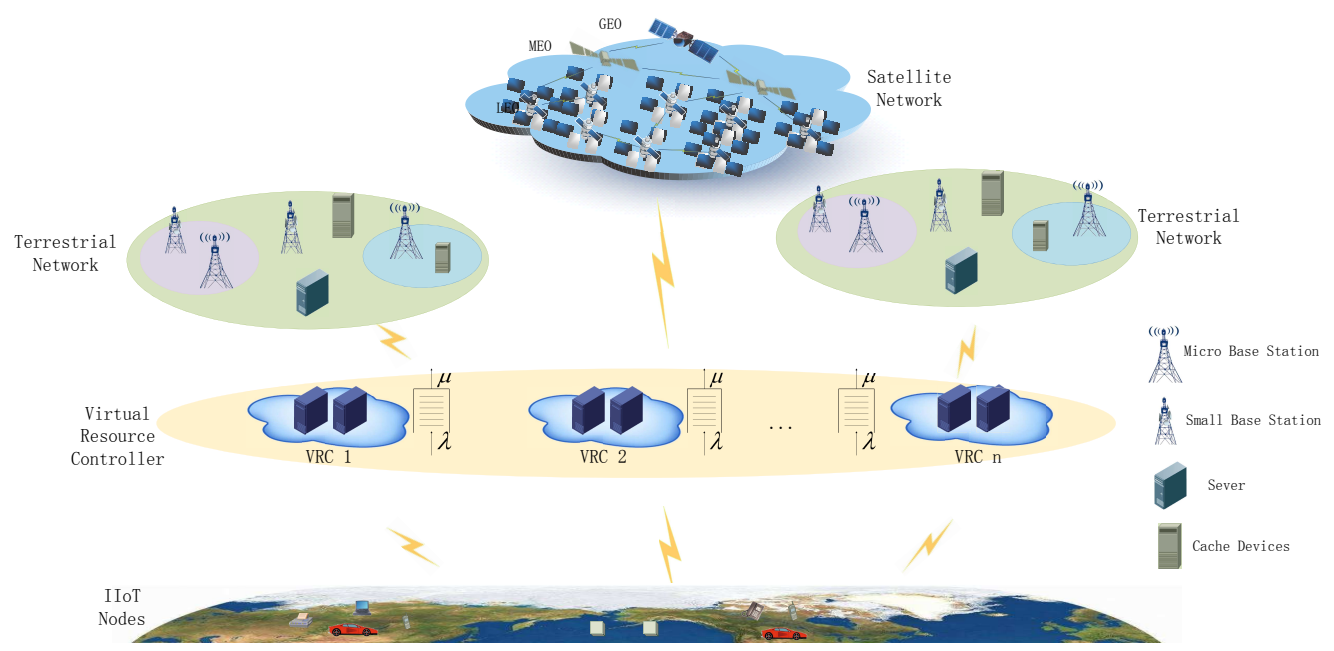

Fig. 1. The transoceanic logistics in the integrated satellite-terrestrial network

\section{System ARCHitecture}

The application scenario considered is transoceanic logistics, and the architecture is illustrated in Fig. 1. It contains IIoT nodes in transoceanic logistics, virtual resource controller (VRC), the terrestrial networks (TN) and the satellite networks (SN). The satellite networks consist of the LEO, the MEO and the GEO satellites. And based on the idea of SDN, LEO is responsible for data forwarding as the data side in the satellite network, while MEO and GEO play roles as SDN controllers in much circumstances, but they can also undertake data forwarding tasks according to the requirements of IIoT node when necessary. In order to obtain better performances, the whole architecture adopts a hierarchical architecture. To be simple, we put $\mathrm{SN}$ and TN together as service sides.

In this system, sensors are responsible for collecting data from IIoT nodes, VRCs are responsible for selecting service sides for IIoT nodes according to the requirements of IIoT nodes and the number of different types of resources owned by service sides. And VRCs are also responsible for establishing the connection between IIoT node and base stations or satellites. Each terrestrial or satellite node contains computation, storage and communication resources. Different terrestrial or satellite nodes have different resource configurations, i.e., the amount of different types of resources are different in different terrestrial or satellite nodes. In each VRC, we use the $\mathrm{M} / \mathrm{M} / 1$ queuing model to analyze resource utilization and system service delay at different arrival rate $\lambda$ and service rate $\mu$.

The detailed work process is depicted as follows.

- Since the application scenario under consideration is transoceanic logistics, the distribution of IIoT nodes will be particularly extensive. In the case of broad range of distribution, IIoT nodes may exceed the coverage of the terrestrial networks. Due to the broader coverage of the satellite networks, when IIoT nodes are located at sea or remote areas that outside the coverage of the terrestrial networks, the satellite networks can maintain the service and provide a seamless connection service for IIoT nodes in the transoceanic logistics.

- After sensors in IIoT nodes collect information including delay, amount of data to be transmitted, and the preference of resources types, etc., the information is uploaded to VRC. For IIoT nodes have different types of traffic requirements, and they are distributed in different places, VRC is responsible for determining the different requirements of different IIoT nodes and for determining the number of computation, storage and communication resources for IIoT nodes based on the information.

- After the information uploaded to VRC, VRC determines the number of resource cubes required based on the received information. Then it matches IIoT nodes with service sides based on MCPR algorithm. And according to different requirements of resources, VRC chooses different service sides to provide service for different IIoT nodes.

- After determining the service side of IIoT node, the total amount of resources required by all IIoT nodes served by each $\mathrm{TN}$ or $\mathrm{SN}$ is also determined. In this case, resources of some TN or SN are insufficient while resources of some $\mathrm{TN}$ or $\mathrm{SN}$ are remaining. If resources of $\mathrm{TN}$ or $\mathrm{SN}$ cannot meet requirements of IIoT nodes it served, it will bring about unnecessary waiting delay. Therefore, we design an algorithm, MCRC algorithm, to adjust matching results under the consideration of resource cubes. And the adjustment is also accomplished by VRC.

For obtaining higher resource utilization and smarter connections, we consider the preference of IIoT nodes for resource tpes in the designed matching algorithm: MCPR algorithm. 
As mentioned above, VRC plays an important role in the whole process. Like a link, VRC matches IIoT nodes and network service sides when IIoT nodes and service sides are not in contact.

TABLE I

SYMBOL COMPARISON

\begin{tabular}{cc}
\hline Symbol & Definition \\
\hline$U$ & The set of IIoT nodes \\
$u_{j}$ & IIoT node \\
$U_{j}$ & The task of node $u_{j}$ \\
$o_{j}$ & The total delay \\
$l_{j}$ & The amount of data \\
$\Gamma_{j}$ & The resource requirements of node $u_{j}$ \\
$a_{j}$ & The allocation of different resources \\
$q_{j}$ & Queuing delay \\
$t_{j}$ & Propagation delay \\
$f_{j}$ & Computing delay \\
$l_{n j}$ & The distance between $u_{j}$ and service side \\
$\psi=(g, \mathbf{a})$ & One kind of network resource configuration \\
$L_{j}^{u t}, L_{n}^{t u}, L_{m}^{s u}$ & Preference Lists \\
\hline
\end{tabular}

\section{Resource Cube And Problem Formulation}

For ease of understanding, the main symbol definitions used are listed in table I. We use $U_{j}=\left\{o_{j}, l_{j}, \Gamma_{j}\right\}$ to describe the task of node $u_{j}$ needs to accomplish. Among these definitions, $o_{j}$ is the total delay, $l_{j}$ is the amount of data to be processed, and $\Gamma_{j}$ is a vector consisting of the requirements of $u_{j}$ for three kinds of resources, i.e. $\Gamma_{j}=\left\{\Gamma_{j}^{c m}, \Gamma_{j}^{c p}, \Gamma_{j}^{s t}\right\}$.

For the $n$th terrestrial node, we use $t_{n}^{s t} t_{n}^{c p}$ and $t_{n}^{c m}$ to denote the storage resource, the computation resource, and the communication resource, respectively. While for the $n$th satellite node, we use $s_{n}^{s t} s_{n}^{c p}$ and $s_{n}^{c m}$ to denote the storage resource, the computation resource, and the communication resource, respectively.

It is well known that transoceanic logistics in the IIoT is characterized by massive connections, and data volume of each connection is very small. Therefore, in order to realize flexible allocation of multi-dimensional resources, we design the resource cube, as shown in Fig. 2. The unit resource cube is composed of one portion communication resource, one portion computation resource and one portion of storage resource.

Considering the characteristics of transoceanic logistics in the IIoT, we can easily get that the amount of data of each IIoT node is small. Then the physical resources contained in the portions of various resources are defined as follows: one portion of communication resources refers to $1 \mathrm{kbit} / \mathrm{s}$ bandwidth, denoted as $b$; one portion of computation resource refers to one CPU cycle per second, which one CPU cycle per second can deal with $1 \mathrm{kbits}$ data, denoted as $c$; and one portion of storage resource refers to storage space of $1 \mathrm{kbits}$ data, denoted as $s$.

With the resource cube definition in Fig. 2, we use a vector $a_{j}$ to describe the allocation of different resources. For $a_{j}=\left\{a_{j}^{b}, a_{j}^{c}, a_{j}^{s}\right\}$, where $a_{j}^{b}, a_{j}^{c}$ and $a_{j}^{s}$ are the

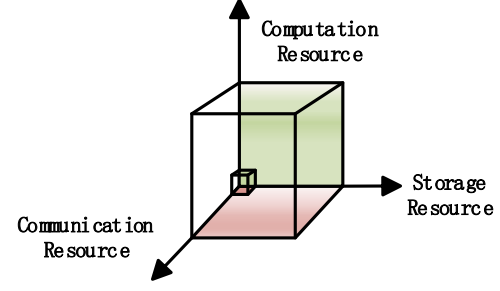

Fig. 2. The sample of the resource cube

amount of communication, computation and storage resources contained in $a_{j}$ that allocated to the IIoT node $u_{j}$ respectively. After given the number of different resources allocated to the IIoT node $u_{j}$, the ability of resource cubes allocated to the IIoT node $u_{j}$ is determined accordingly.

In this paper, we adopt service delay to evaluate the QoS. For node $u_{j}, \forall u_{j} \in U$, service delay is denoted as $o_{j}=q_{j}+t_{j}+f_{j}$, where $U$ represents the set of IIoT nodes, $q_{j}$ is queuing delay, $t_{j}$ is propagation delay, and $f_{j}$ is computing delay. Assuming that the workload of each IIoT node $u_{j}$ obeys the Poisson arrival process, based on the M/M/1 queuing model and the allocated resource cube, queuing delay when serving the node $u_{j}$ is $q_{j}=\frac{\lambda}{a_{j}^{b} \mu} \frac{1}{\mu-\frac{\lambda}{a_{j}^{b}}}$. We define the distance between the IIoT node $u_{j}$ and the TN $t_{n}$ as $l_{n j}^{t}$, and similarly, the distance between the IIoT node $u_{j}$ and the $\mathrm{SN} s_{m}$ as $l_{m j}^{s}$. To simplify, we use $l_{n j}$ to denote the distance between the IIoT node $u_{j}$ and service side. Therefore, the propagation delay $t_{j}$ usually obeys $t_{j}=\theta l_{n j} ; \theta$ is a scalar representing the weight factor. Assuming that processing $l_{j}$ data needs $c$ CPU cycles, then we can get that $f_{j}=\frac{l_{j}}{a_{j}^{c} c}$. Then service delay of $u_{j}$ can be expressed as

$$
\begin{aligned}
o_{j} & =q_{j}+t_{j}+f_{j} \\
& =\frac{\lambda}{a_{j}^{b} \mu} \frac{1}{\mu-\frac{\lambda}{a_{j}^{b}}}+\theta l_{n j}+\frac{l_{j}}{a_{j}^{c} c} .
\end{aligned}
$$

The constraint of delay can be written as

$$
\mathrm{C} 1: o_{j} \leq o_{\mathrm{thr}}
$$

where $o_{\mathrm{thr}}$ is the predefined threshold of service delay.

To meet the requirements of $\Gamma_{j}^{r}$ and allocate resources easily, and ensure pulling the job off, resource cubes allocated to IIoT node $u_{j}$ needs to meet the following constraint:

$$
\begin{aligned}
C 2: & a_{j}^{b} b+a_{j}^{c} c+a_{j}^{s} s \geq \Gamma_{j}^{c m}+\Gamma_{j}^{c p}+\Gamma_{j}^{s t} \\
& a_{j}^{b}=a_{j}^{c}\left\|a_{j}^{b}=a_{j}^{s}\right\| a_{j}^{c}=a_{j}^{s} \\
& a_{j}^{b} b+a_{j}^{c} c+a_{j}^{s} s \geq l_{j},
\end{aligned}
$$

i.e., the sum of resources allocated to the IIoT node $u_{j}$ should be greater than the sum of required resources, and the number of any two resources allocated to it is equal considering the convenience of the allocation of resource 
cube. And the number of resource cubes allocated is an integer.

According to the constraint $\mathrm{C} 1$, in order to satisfy the physical meaning of $q_{j}$, value of $a_{j}^{b}$ needs to satisfy that $a_{j}^{b}>\frac{\lambda_{j}}{\mu}$, therefore, we can get an initial value of $a_{j}^{b}$. As long as we make sure that this basic condition is met, the amount of resources allocated to the IIoT node $u_{j}$ can be adjusted without exceeding the amount of resources of its service side. For example, if $\Gamma_{j}=\left\{\Gamma_{j}^{c m}, \Gamma_{j}^{c p}, \Gamma_{j}^{s t}\right\}=$ $\{2,2,4\}$ while the initial value of $a_{j}^{b}$ is 3 , and the storage resource of service side is insufficient, then the allocation can be $a_{j}=\left\{a_{j}^{b}, a_{j}^{c}, a_{j}^{s}\right\}=\{3,3,2\}$. This is just one example, and the final resource allocation is determined according to matching results and resource constraints.

Under constraints $\mathrm{C} 1$ and $\mathrm{C} 2$, it can be concluded that it is unnecessary to allocate resources according to the amount of various resources required by the IIoT node $u_{j}$ absolutely. As long as the total amount of resources allocated exceeds the total amount of resources required by the IIoT node $u_{j}$, and the task processing requirements can be met under the condition of delay tolerance $o_{\mathrm{thr}}$. This means that if the number of resources required by users conflicts with the number of resources existing in the network, i.e., when the number of certain type of resource in the network is insufficient, other types of resources can be used to replace it under certain conditions. Let give a special example to clarify the replacement:

$$
\begin{aligned}
o_{j}^{1} & =\frac{\lambda}{a_{j}^{b, 1} \mu} \frac{1}{\mu-\frac{\lambda}{a_{j}^{b, 1}}}+\theta l_{n j}+\frac{l_{j}}{a_{j}^{c, 1} c} \\
& =\frac{\lambda}{a_{j}^{b, 2} \mu} \frac{1}{\mu-\frac{\lambda}{a_{j}^{b, 2}}}+\theta l_{n j}+\frac{l_{j}}{a_{j}^{c, 2} c}=o_{j}^{2},
\end{aligned}
$$

where $a_{j}^{b, 1}$ and $a_{j}^{c, 1}, a_{j}^{b, 2}$ and $a_{j}^{c, 2}$ represents the different amount of communication and computation resources contained in two different resource cubes $a_{j}^{1}$ and $a_{j}^{2}$.

As a result, there may be several possible ways of allocating that meets constraints $\mathrm{C} 1$ and $\mathrm{C} 2$. Therefore, how to choose the most appropriate way from multiple resource allocation ways is the problem that needs to be dealt with. The resource allocation we choose takes into account both the resource requirements of the IIoT node $u_{j}$, i.e. $\Gamma_{j}$, and and the number of resources on the service side (TN or $\mathrm{SN}$ ). For ease of resource allocation, the value of $a_{j}^{b}, a_{j}^{c}$, and $a_{j}^{s}$ start at one and increase by one.

We can get the constraint of resource cubes as follows:

$$
\begin{aligned}
C 3: \sum_{j \in U} a_{j}^{b} b & \leq \sum_{n \in T} t_{n}^{c m}+\sum_{m \in S} s_{m}^{c m} \\
\sum_{j \in U} a_{j}^{c} c & \leq \sum_{n \in T} t_{n}^{c p}+\sum_{m \in S} s_{m}^{c p} \\
\sum_{j \in U} a_{j}^{s} s & \leq \sum_{n \in T} t_{n}^{s t}+\sum_{m \in S} s_{m}^{s t},
\end{aligned}
$$

i.e., the total number of resource cubes assigned to IIoT nodes cannot exceed the total amount of resources of the terrestrial and the satellite networks.

Constraint C3 can be divided into two parts based on services provided by different networks, as shown below. If IIoT nodes are served by the satellite networks, which are denoted as $U^{S}$, then constraint C3 can be simplified as

$$
\begin{aligned}
C 3^{S}: \sum_{j \in U^{S}} a_{j}^{b, S} b & \leq \sum_{m \in S} s_{m}^{c m} \\
\sum_{j \in U^{S}} a_{j}^{c, S} c & \leq \sum_{m \in S} s_{m}^{c p} \\
\sum_{j \in U^{S}} a_{j}^{s, S} s & \leq \sum_{m \in S} s_{m}^{s t} .
\end{aligned}
$$

Similarly, if it is served by the terrestrial networks, constraint $\mathrm{C} 3$ can be simplified as

$$
\begin{aligned}
C 3^{T}: & \sum_{j \in U^{T}} a_{j}^{b, T} b \leq \sum_{n \in T} t_{n}^{c m} \\
\sum_{j \in U^{T}} a_{j}^{c, T} c & \leq \sum_{n \in T} t_{n}^{c p} \\
\sum_{j \in U^{T}} a_{j}^{s, T} s & \leq \sum_{n \in T} t_{n}^{s t},
\end{aligned}
$$

where $U^{T}$ is used to denote IIoT nodes served by the terrestrial networks.

We use $x_{j n}^{t}$ and $x_{j m}^{s}$ as the indicator to describe whether IIoT nodes is connected to $\mathrm{TN} t_{n}$ or $\mathrm{SN} s_{m}$, and the value of this indicator is 1 when connected. We can get the constraint $\mathrm{C} 3$ as:

$$
C 3: \sum_{n \in T} x_{j n}^{t}+\sum_{m \in S} x_{j m}^{s}=1, \quad \forall j \in J .
$$

In order to simplify, we set $g=\sum_{n \in T} x_{j n}^{t}$, then $\sum_{m \in S} x_{j m}^{s}=1-g$. Therefore, we can get that $g$ is a binary variable: $g \in\{0,1\}$. Then $\mathrm{C} 3$ can be converted to

$$
C 3: g \in\{0,1\} \text {. }
$$

The ultimate goal is to achieve the highest resource utilization while meeting requirements $U_{j}\left(o_{t h r}, l_{j}\right)$, therefore, we define the utility function as:

$$
R\left(g, a_{j}\right)=\sum_{n \in N^{T}, m \in M^{S}} \sum_{j \in U}\left[g F_{j}^{N^{T}}+(1-g) F_{j}^{M^{S}}\right]
$$

where $F_{j}^{N^{T}}$ and $F_{j}^{M^{S}}$ are the utilization of resource cubes of the terrestrial and the satellite networks respectively, i.e.,

$$
\begin{aligned}
& F_{j}^{N^{T}}=\frac{a_{j}^{b} b+a_{j}^{c} c+a_{j}^{s}}{t_{n}^{c p}+t_{n}^{c m}+t_{n}^{s t}} \\
& F_{j}^{M^{S}}=\frac{a_{j}^{b} b+a_{j}^{c} c+a_{j}^{s} s}{s_{m}^{c p}+s_{m}^{c m}+s_{m}^{s t}} .
\end{aligned}
$$


Then the problem can be written as:

$$
\begin{array}{ll} 
& \max _{g, a_{j}} R\left(g, a_{j}\right) \\
\text { s.t. } & o_{j} \leq o_{t h r}, \forall j \in U, \\
& a_{j}^{b} b+a_{j}^{c} c+a_{j}^{s} s \geq \Gamma_{j}^{c m, r}+\Gamma_{j}^{c p, r}+\Gamma_{j}^{s t}, \\
& a_{j}^{b}=a_{j}^{c}\left\|a_{j}^{b}=a_{j}^{s}\right\| a_{j}^{c}=a_{j}^{s}, \\
& a_{j}^{b} b+a_{j}^{c} c+a_{j}^{s} s \geq l_{j}, \\
& \sum_{j \in U} a_{j}^{b} b \leq \sum_{n \in T} t_{n}^{c m}+\sum_{m \in S} s_{m}^{c m}, \\
& \sum_{j \in U} a_{j}^{c} c \leq \sum_{n \in T} t_{n}^{c p}+\sum_{m \in S} s_{m}^{c p}, \\
& \sum_{j \in U} a_{j}^{s} s \leq \sum_{n \in T} t_{n}^{s t}+\sum_{m \in S} s_{m}^{s t}, \\
& g \in\{0,1\} .
\end{array}
$$

For further consideration, since the allocation of the number of resource cubes can only be integer, the above optimization problem is an integer and non-convex problem. Considering the large number of nodes, it is difficult to solve. Therefore, in order to get optimal solution, we will adopt Markov chain and Morkov approximation. And to improve the utility of the whole system and determine which network node to provide services, we will adopt a matching game between IIoT nodes and TN or SN, which is presented in section IV.

\section{Matching GAME AND Markov ApProximation}

\section{A. Markov Approximation and Markov Chain of Resource} Cubes

In order to deal with the integer and non-convex problems (9), in this section, we will use Markov approximation to convert (9) into a solvable form, and then Markov chain is adopted to depict the state transitions of resource cubes, and to get the optimal distribution probability.

1) Markov Approximation: As described above, the set of nodes is $U$, and the network resource configuration set that satisfies the constraint can be defined as $\Psi$. For the sake of simplicity, we let $R(g, \mathbf{a})=R_{\psi}$, where $\psi=(g, \mathbf{a})$ is one kind of network resource configuration. Therefore, we can get the objective function:

$$
\max _{\psi \in \Psi} R_{\psi} .
$$

However, $R_{\psi}$ is unsolvable. Hence, based on Markov approximation, by introducing $p_{\psi}$, it is transformed into equivalent function:

$$
\begin{aligned}
& \max _{p \geq 0} \sum_{\psi \in \Psi} p_{\psi} R_{\psi} \\
\text { s.t. } & \sum_{\psi \in \Psi} p_{\psi}=1,
\end{aligned}
$$

where $p_{\psi}$ is the probability of choosing the network resource configuration $\psi$. And as proved and introduced in [32] [33], the optimal solution of (10) and (11) is the same. But due to the coupling nature between variables, the solution of (11) is also difficult to get. Then we will take the log-sum-exp approximation further to solve the problem.

As the log-sum-exp approximation is a closed and convex function, we can get a differentiable approximation function as:

$$
\max _{\psi \in \Psi} R_{\psi}=\frac{1}{\zeta} \log \left[\sum_{\psi \in \Psi} \exp \left(\zeta R_{\psi}\right)\right] \approx g_{\zeta}\left(R_{\psi}\right),
$$

where $\zeta$ is a positive constant. And the accuracy of the approximation is

$$
0 \leq\left|R_{\max }-g_{\zeta}\left(R_{\psi}\right)\right| \leq \frac{1}{\zeta} \log \Psi,
$$

where as $\zeta \rightarrow \infty, \frac{1}{\zeta} \log \Psi \rightarrow 0 \varsigma \rightarrow \infty$, and the specific mathematical derivation process can be found in [33]. As a result, we can get a equivalent problem of (11), i.e.,

$$
\begin{array}{ll}
\max _{p \geq 0} & \sum_{\psi \in \Psi} p_{\psi} R_{\psi}-\frac{1}{\zeta} \sum_{\psi \in \Psi} p_{\psi} \log p_{\psi} \\
\text { s.t. } & \sum_{\psi \in \Psi} p_{\psi}=1 .
\end{array}
$$

Then by solving the KKT condition of problem (14), we can get the optimal probability distribution $p^{\circ}$ :

$$
\begin{aligned}
p_{\psi}^{\circ}\left(R_{\psi}\right) & =\frac{\exp \left(\zeta R_{\psi}\right)}{\sum_{\psi^{\circ} \in \Psi} \exp \left(\zeta R_{\psi^{\circ}}\right)} \\
& =\frac{1}{\sum_{\psi^{\circ} \in \Psi} \exp \left(\zeta\left(R_{\psi^{\circ}}-R_{\psi}\right)\right)} .
\end{aligned}
$$

However, the solution of (15) is difficult to get because it requires complete information about all possible configurations, but this information is beyond the reach of a single VRC. Hence, we adopt the probability obtained by Markov chainto replace $p_{\psi}^{\circ}$, for the difference is also bounded.

2) Markov Chain of Resource Cubes: According to the definition of resource cubes, it can determine the corresponding ability. Then VRC can match the number of resource cubes with the arrival tasks. And after the task is completed, resource cubes that it used will be released and reassigned to other tasks.

As mentioned in section III, within a VRC, it can be seen as a $\mathrm{M} / \mathrm{M} / 1$ queuing model with service rate $\mu$ and arrival rate $\lambda$. Then after the description of resource cubes as a foreshadowing, we can also get the total number of available resource cubes when given TNs or SNs that can be accessed within a VRC. Therefore, after defining resource cubes, the change of resource cubes within a 
VRC can also be determined. Assume that the change in the state of the resource cubes occurs only at the beginning of the interval, then it can be described as follows:

- Assuming that at the beginning of the time interval, the total number of the available resource cubes is $N$, and the first arrival task requires $m$ resource cubes, then the number of resource cubes change to $N-m$, with the probability of $p_{N, N-m}$.

- Assuming that at the beginning of the time interval, there are $k$ resource cubes, while $m$ resource cubes are released, then the number of resource cubes is changed from $k$ to $k+m$, with the probability of $p_{k, k+m}$.

- As we all know, satellites move continuously, thus causing changes in its coverage. As a result, assuming that with the movement of satellites, at the beginning of the time interval, a satellite with $k$ resource cubes cannot provide service, and at the end of the former time interval the number of resource cubes is $m$, then the state of Markov chain changes from $m$ to $m-k$, with the probability of $p_{m, m-k}$. Therefore, as an inverse process, the state of Markov chain may change from $m$ to $k+m$ with the probability of $p_{m, k+m}$, as a satellite with $k$ resource cubes can provide service at the beginning of the time interval.

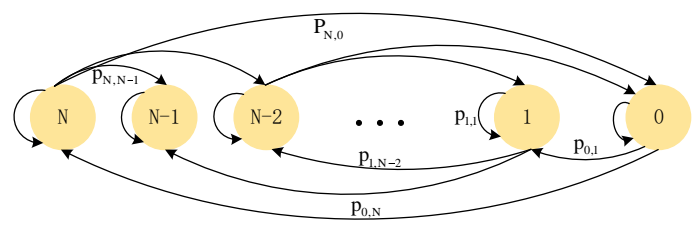

Fig. 3. Markov chain of the resource cube state transition

Thus we can get the state transition diagram of resource cubes, as shown in Fig. 3. To simplify the system, we assume that the resource cube changes at a fixed interval, and assume that the fixed interval is $\Delta t$, and $\Delta t=$ $(k \delta,(k+1) \delta]$. As we adopt the $\mathrm{M} / \mathrm{M} / 1$ queuing model to analyze the small system within a VRC, we can compute the distribution possibility based on the characteristics of $\mathrm{M} / \mathrm{M} / 1$. In order to get the probability, we first give some definitions as follows:

$N$ : the total number of resource cubes;

$n$ : the total number of the required resource cubes of arrival tasks at the beginning of the time interval;

$\lambda$ : the arrival rate of the task of IIoT nodes;

$q_{a}$ : the probability that a new task arrive before the next time interval begin;

$q_{f}$ : the probability that a task finish before the next time interval begin;

$Q_{p}(i, n)$ : the probability that $i$ resource cubes are required among $N-n$ available resource cubes in current time interval;

$Q_{f}(i, n)$ : the probability that $i$ resource cubes are released among $n$ occupied resource cubes.
After calculation we can get $q_{a}=1-e^{-\lambda}$ and $q_{f}=$ $q_{a}{ }^{-1}$. If $n$ is given, we can get:

$$
\begin{gathered}
Q_{p}(i, n)=\left(\begin{array}{c}
N-n \\
i
\end{array}\right)\left(1-q_{a}\right)^{N-n-i} q_{a}^{i}, \\
Q_{f}(i, n)=\left(\begin{array}{c}
n \\
i
\end{array}\right)\left(1-q_{f}\right)^{n-i} q_{f}^{i} .
\end{gathered}
$$

As shown in Fig. 3, $p_{n, n+i}$ denotes the probability that there is $n$ resource cubes at the beginning of the time interval and $n+i$ resource cubes at the beginning of the next time interval; and $p_{n+i, n}$ denotes the probability that there is $n+i$ resource cubes at the beginning of the time interval while $i$ resource cubes are used. Therefore, we can get the state transition probability:

$$
p_{n, n+i}=Q_{p}(\chi, n) Q_{f}(\chi+i, n), 1 \leq i \leq n,
$$

$p_{n, n-i}=Q_{p}(\chi+i, n) Q_{f}(\chi, n), 0 \leq i \leq N-n-\chi$,

where $\chi$ is the number of resource cubes previously occupied or released, respectively.

Then we can get more detailed equations as follows:

$$
\begin{aligned}
p_{n, n+i} & =\left(\begin{array}{c}
N-n \\
\chi
\end{array}\right)\left(\begin{array}{c}
n \\
\chi+i
\end{array}\right) \frac{\left(1-q_{a}\right)^{N-2 n+i}}{q_{a}{ }^{i}}, \\
p_{n, n-i} & =\left(\begin{array}{c}
N-n \\
\chi+i
\end{array}\right)\left(\begin{array}{c}
n \\
\chi
\end{array}\right)\left(1-q_{a}\right)^{N-2 n+i} q_{a}{ }^{i} .
\end{aligned}
$$

At this point, all variables VRC are known, i.e. $p_{n, n+i}$ and $p_{n, n+i}$ can get specific values. And we plug this probability into the utility formula and decide whether to choose $\mathrm{TN}$ or $\mathrm{SN}$ as the service provider. In the next section, we will deploy matching games between network nodes and IIoT nodes, and determine the specific service side for each IIoT node.

\section{B. Matching Game between IIoT Nodes and TN or SN}

As mentioned above, we adopt several M/M/1 queuing models to describe the system. And we use matching games to match IIoT nodes with network node of TN or SN. As a result, in each VRC, TN or SN provides service for a part of IIoT nodes, and it can be seen as an independent system.

In the designed STN architecture, VRC is responsible for matching IIoT nodes to TNs or SNs. Within a VRC, there is a many-to-many matching between IIoT nodes and TNs or SNs. When it has been determined which network node of $\mathrm{TN}$ or $\mathrm{SN}$ is to provide service by the 
distribution probability in the last section, a many-to-many matching is also derived. Assuming that $\mathrm{TN}$ is service side, the preference list of the IIoT node $u_{j}, \forall j \in U^{T}$ about all TNs is $L_{j}^{u t}=\left[L_{j 1}^{u t}, \ldots, L_{j N}^{u t}\right]$, satisfying

$$
L_{j n}^{u t}=\frac{1}{l_{j n}}, \quad \forall n \in T,
$$

where $l_{j n}$ is the distance between the IIoT node $u_{j}$ and the TN $t_{n}$, and $U^{T}$ denotes the set of IIoT nodes served by TNs.

Similarly, the preference list of IIoT nodes $u_{j}, \forall j \in U^{S}$ can be presented as

$$
L_{j m}^{u s}=\frac{1}{l_{j m}}, \quad \forall m \in S
$$

Correspondingly, we set a preference list $L_{n}^{t u}=$ $\left[L_{n 1}^{t u}, L_{n 2}^{t u}, \ldots, L_{n J}^{t u}\right]$ of TN $t_{n}, \forall n \in T$, over all IIoT nodes, i.e.,

$$
L_{j n}^{t u}=\alpha \frac{t_{n}^{c m}}{a_{j}^{b} b}+\beta \frac{t_{n}^{c p}}{a_{j}^{c} c}+\gamma \frac{t_{n}^{s t}}{a_{j}^{s} s},
$$

and the definition of the preference list $L_{m}^{s u}=$ $\left[L_{m 1}^{s u}, L_{m 2}^{s u}, \ldots, L_{m J}^{s u}\right]$ of $\mathrm{SN} s_{m}, \forall m \in S$ over all IIoT nodes are similar to $\mathrm{TN}$,

$$
L_{j m}^{s u}=\alpha \frac{s_{m}^{c m}}{a_{j}^{b} b}+\beta \frac{s_{m}^{c p}}{a_{j}^{c} c}+\gamma \frac{s_{m}^{s t}}{a_{j}^{s} s},
$$

where $\alpha, \beta$ and $\gamma$ are weight factors, which are relevant to requirements of IIoT nodes, and meet $[\alpha, \beta, \gamma]=$ $\left[\frac{\Gamma_{j}^{c m, r}}{\Gamma_{j}^{c m, r}+\Gamma_{j}^{c p, r}+\Gamma_{j}^{s t, r}}, \frac{\Gamma_{j}^{c p, r}}{\Gamma_{j}^{c m, r}+\Gamma_{j}^{c p, r}+\Gamma_{j}^{s t, r}}, \frac{\Gamma_{j}^{s t, r}}{\Gamma_{j}^{c m, r}+\Gamma_{j}^{c p, r}+\Gamma_{j}^{s t, r}}\right]$.

As a result, based on preference lists $L_{j}^{u t}$ and $L_{n}^{t u}$ or $L_{m}^{s u}$, there exists a many-to-many matching algorithm for TN-IIoT or SN-IIoT pairing problem, which is presented in Algorithm 1: matching considering preference (MCPR) algorithm.x

After the many-to-many matching is derived, within a VRC, IIoT nodes are initially matched to network nodes based on resource preferences. However, these matching results ignore the fact that the service sides may have insufficient resources, so we change some of the matching results.

According to the resource utilization optimization problem solved by Markov approximation and Markov chain, we can adjust the matching results of some IIoT nodes that TNs or SNs can hardly meet its demand, and steps are shown in Algorithm 2: matching considering resource cubes (MCRC) algorithm.

After the Algorithm2: MCRC algorithm, we will adjust matching network node for some of the IIoT nodes. By doing so, the service delay can be reduced significantly, which are presented in the next section.

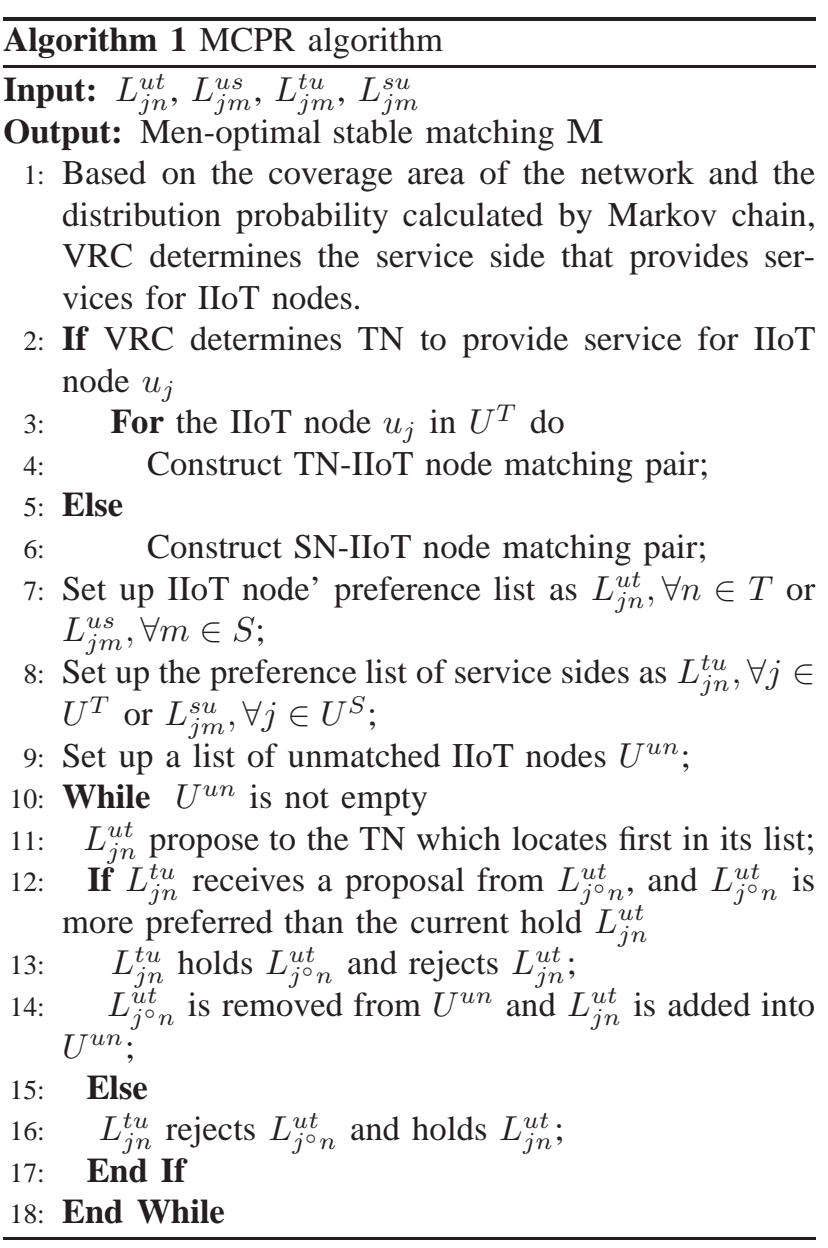

\section{Simulation Results}

In this section, we will present the performance of the proposed MCRC algorithm and MCPR algorithm comparing them with the random selection way and the Hungary assignment algorithm [35]. The simulation scenario includes satellite networks consisting of one GEO, two MEO and three LEO satellites and the terrestrial networks consisting of 15 base stations. The altitudes of the LEO, the MEO and the GEO satellites are $887 \mathrm{~km}$, $2000 \mathrm{~km}$ and $35786 \mathrm{~km}$, respectively. The number of IIoT nodes is set to 400 . The delay tolerance of TN and SN are $10 \mathrm{~ms}$ and $740 \mathrm{~ms}$. Service rate of each resource cube is represented by $\mu$, with unit arrival rate is represented by $\lambda$.

In the case of satellites as service sides, taking the GEO satellites with highest time delay as an example to explain the set of delay tolerance. The GEO satellites are $35786 \mathrm{~km}$ away from the ground and the data transmission speed is set to $50 \mathrm{~km} / \mathrm{ms}$ [12]. Then only data propagation delay is $715.72 \mathrm{~ms}$, hence we set the delay tolerance of satellites to $750 \mathrm{~ms}$. However, this is the worst case. The LEO and the MEO satellites are preferred by the preference lists when they can provide services, therefore data transmission delays are not as large. Meanwhile, IIoT nodes served by the GEO satellites are very few while the GEO satellites 


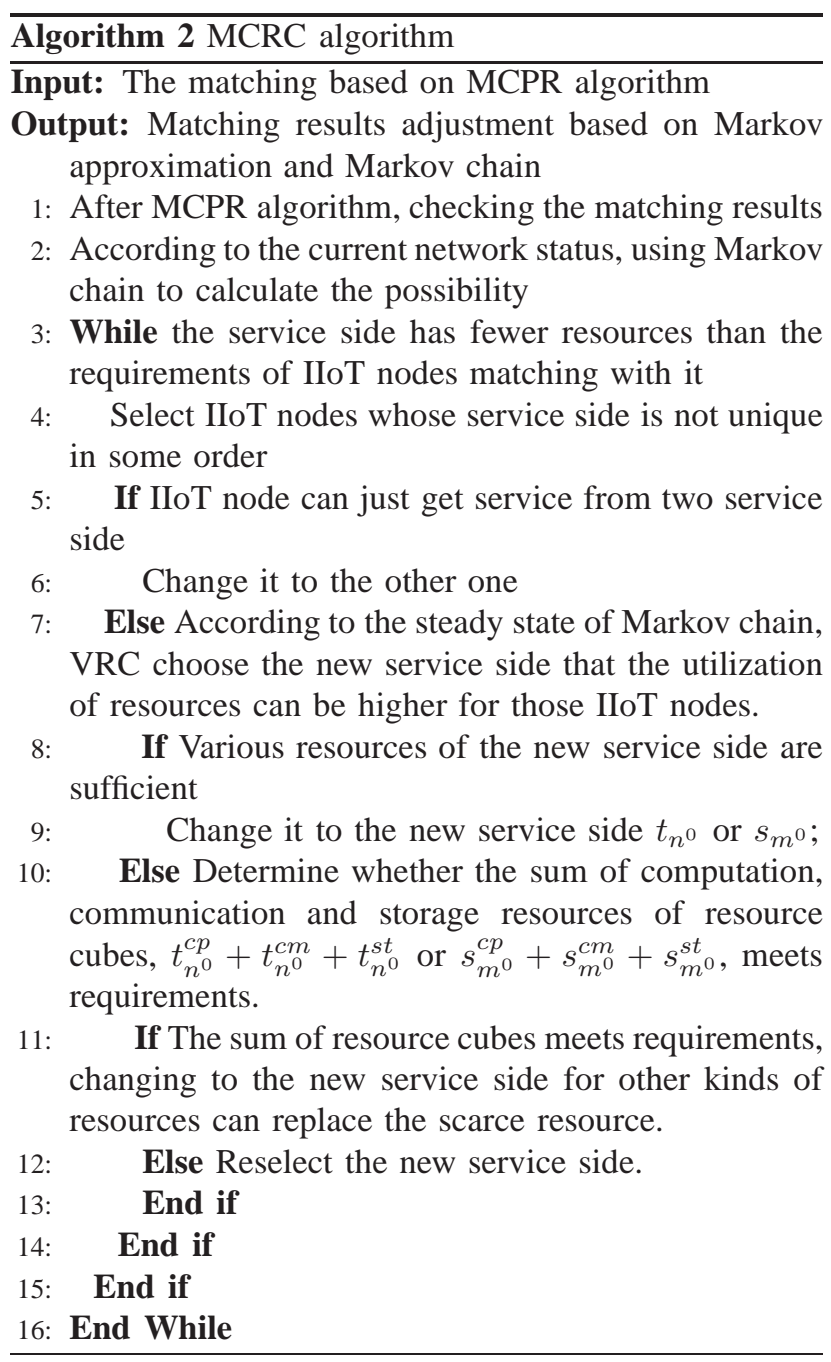

are the last choice.

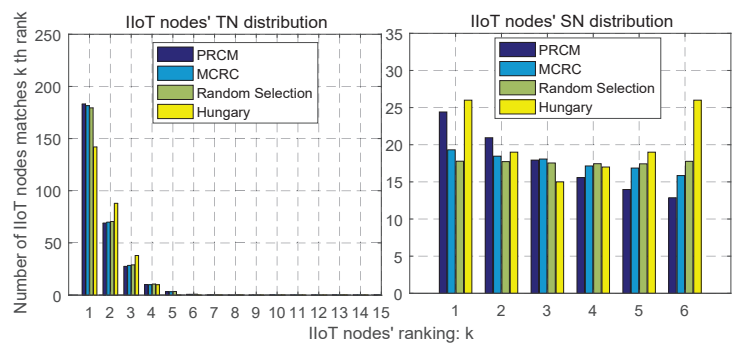

Fig. 4. IIoT nodes' distribution

Meanwhile, in order to eliminate the impact of randomness on algorithm results, we use the mean of 500 runs as final results.

For IIoT nodes, matching results are presented in Fig. 4. As shown, most IIoT nodes can match the first option in its preference list in the MCPR algorithm for these matching results just based on the preference lists of IIoT nodes and network nodes, while it dose not take the amount of resources into account. That matching results may bring

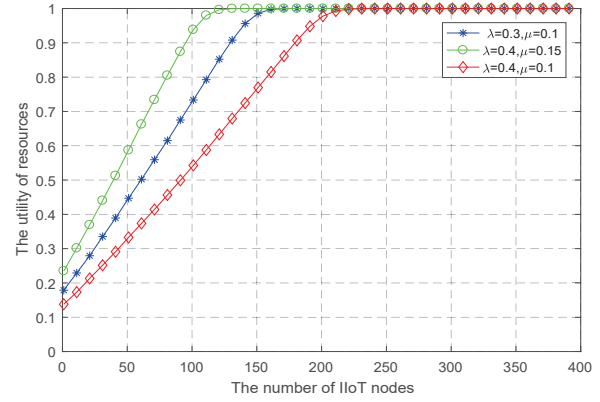

Fig. 5. The comparison of utilization under MCRC

about insufficient resources of some service sides (TN or $\mathrm{SN})$ to meet the needs of all its nodes, and resulting in unnecessary delay: waiting delay of the queueing delay.

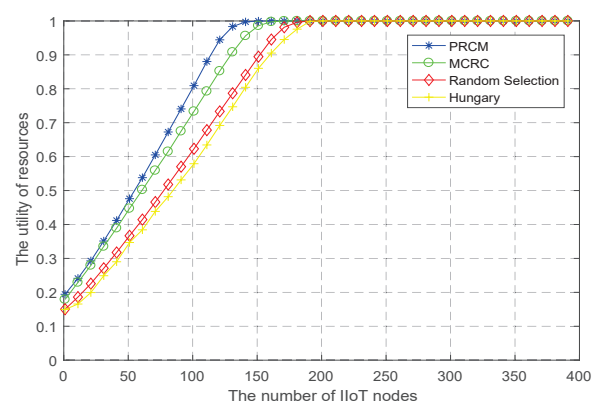

(a) $\mu=0.1 / \mathrm{ms}$ and $\lambda=0.3 / \mathrm{ms}$

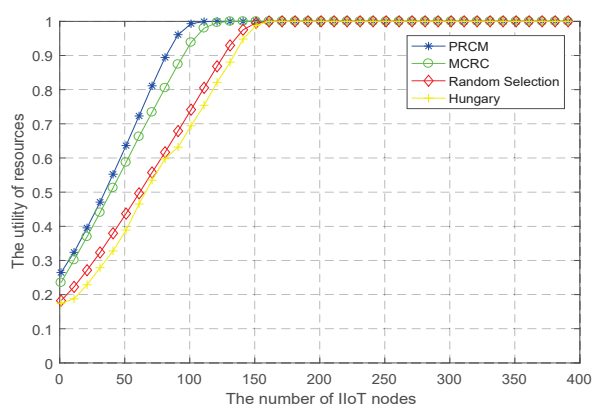

(b) $\mu=0.15 / \mathrm{ms}$ and $\lambda=0.4 / \mathrm{ms}$

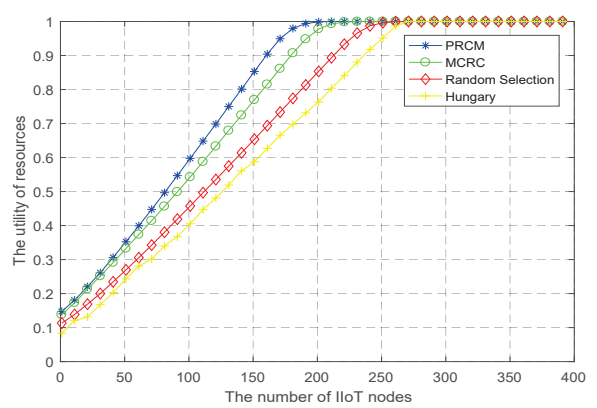

(c) $\mu=0.1 / \mathrm{ms}$ and $\lambda=0.4 / \mathrm{ms}$

Fig. 6. The utilization under different $\mu$ and $\lambda$

Based on above considerations, we choose IIoT nodes whose matching service side (TN or SN) cannot provide sufficient resources, and then adjust the matching result for 
those IIoT nodes. According to MCRC algorithm, these IIoT nodes are matching with other resource-rich service sides. As a result, parts of IIoT nodes match with the service side locates at latter of its preference list, as presented in Fig. 4. Although these IIoT nodes cannot match with the most preferred service side, they match with the service side that can provide sufficient resources to reduce the service delay. And matching results of the random selection way and the Hungary assignment algorithm are also presented in Fig.4 as comparing methods.

As shown in Fig. 4, only a few IIoT nodes change matching results after the MCRC algorithm. However, this adjustment of matching results can minimize unnecessary delay. The reduced delay part is much more significant for a single IIoT node than for the entire system. Although the resource utilization of MCRC algorithm is slightly lower than that of MCPR algorithm due to the change of matching results, the total delay of system of MCRC algorithm is significantly lower than that of MCPR algorithm. Both resource utilization and system delay, performances of the MCPR algorithm and the MCRC algorithm are better than that of the random selection way and the Hungary algorithm.

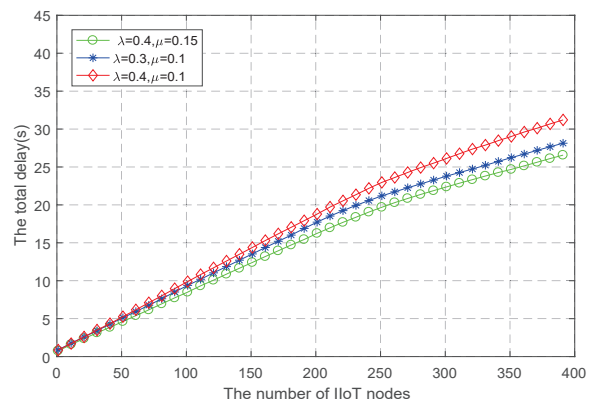

Fig. 7. The comparison of total delay under MCRC

As shown in Fig. 5, we can note that as service rate of each resource cube increases while arrival rate remains the same, the utilization will increase because the processing capacity of the system is improved while the volume of business has not increased; and as arrival rate increases while service rate of each resource cube remains the same, the utilization will decrease because the volume of business increases while the processing capacity of the system remains unchanged. Since the number of resource cubes required by IIoT nodes decreases as arrival rate does not change while service rate of each resource cube increases, it appears that the utilization rate will decrease. And since the number of resource cubes required by IIoT nodes increases as service rate of each resource cube remains the same and arrival rate increases, it appears that the utilization rate will increase.

As shown in Fig. 6, regardless of the combination of different values of $\mu$ and $\lambda$, the resource utilization of the entire system is slightly lower in MCRC algorithm than in MCPR algorithm, and all of these two algorithms have the

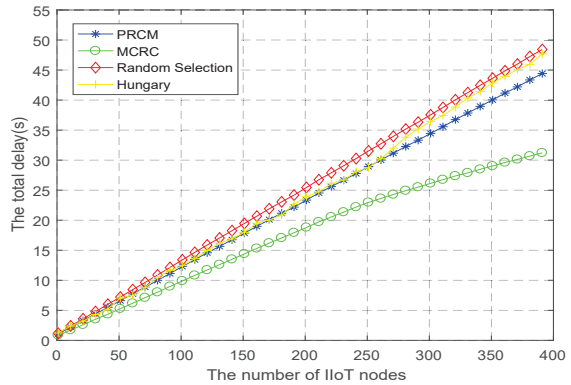

(a) $\mu=0.1 / \mathrm{ms}$ and $\lambda=0.3 / \mathrm{ms}$

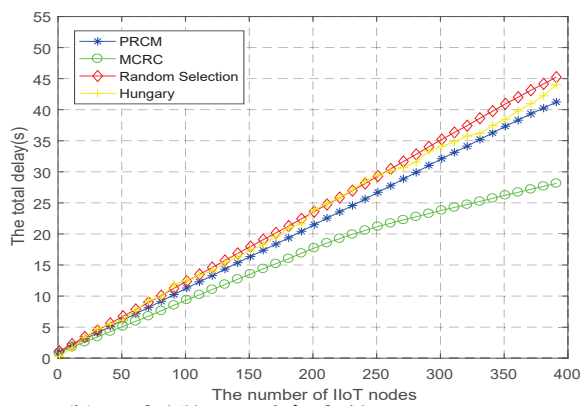

(b) $\mu=0.15 / \mathrm{ms}$ and $\lambda=0.4 / \mathrm{ms}$

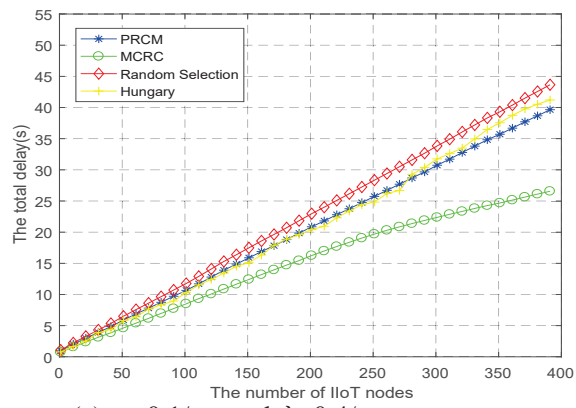

(c) $\mu=0.1 / \mathrm{ms}$ and $\lambda=0.4 / \mathrm{ms}$

Fig. 8. The total delay under different $\mu$ and $\lambda$

better resource utilization performances than the random selection way and the Hungary algorithm. Meanwhile, take $\mu=0.15 / \mathrm{ms}$ and $\lambda=0.4 / \mathrm{ms}$ as an example to illustrate the resource utilization, as presented in Fig. 6(b), the resource utilization in MCPR algorithm is maximized when the number of IIoT nodes is about 110, about 130 in MCRC algorithm, about 150 in the random selection way and about 160 in the Hungary algorithm. This means that the resource allocation of MCPR algorithm, MCRC algorithm, the random selection way and the Hungary algorithm is optimized after the number of IIoT nodes reaches $110,130,150$ and 160 , respectively. The reason is that MCRC algorithm adjusts the matching results on the basis of MCPR algorithm, which is no longer the optimal matching according to resource preference, resulting in the waste of some resources.

As Fig. 7 illustrates, when service rate of each resource cube rises while arrival rate remains unchanged, the total delay of the entire system will decrease because of the increased processing capability of the resource cube. 
When service rate of each resource cube is unchanged while arrival rate increases, the total delay of the system increases for the reason that the traffic volume has been increased. And this result is in line with our common sense.

Fig. 8 illustrates the total delay of the whole system. Again, regardless of the combination of $\mu$ and $\lambda$ values, the results satisfy the analysis: under MCRC algorithm, the total delay is minimized because it reduces the unnecessary waiting delay in the queuing delay, and the random selection yields maximum delay. On one hand, take $\mu=0.15 / \mathrm{ms}$ and $\lambda=0.4 / \mathrm{ms}$ as an example to illustrate the total delay, as presented in Fig. 8(b), values of delay are about $17 \mathrm{~s}, 21 \mathrm{~s}, 24 \mathrm{~s}$ and $24 \mathrm{~s}$ in MCRC algorithm, MCPR algorithm, the random selection way and the Hungary algorithm as the number of IIoT nodes is 200, respectively; while values of delay are about $24 \mathrm{~s}, 32 \mathrm{~s}, 34 \mathrm{~s}$ and $35 \mathrm{~s}$ as the number of IIoT nodes is 300 , respectively. This means that as the number of IIoT nodes increases, the difference becomes larger and larger. On the other hand, for transoceanic logistics, it is not particularly timesensitive, so the total delay in our system can meet requirements.

\section{CONCLUSION}

In this paper, we considered the multi-resource management of IIoT applications, the transoceanic logistics, in integrated terrestrial-satellite networks. In order to achieve the joint management of multi-dimensional resources, we designed a method, resource cube based on virtualization, to describe the granularity of resources. On this basis, we can get requirements of resources of IIoT nodes, and match it with the proper service side. We use MCPR algorithm to do preliminary matching, and then matching results are adjusted according to MCRC algorithm, which take the quantity of resource cubes of service sides and the analysis of Markov approximation into consideration. And for getting more intelligent connectivity, we consider different resource preferences of different IIoT nodes in MCRC algorithm. Although the resource utilization of MCRC algorithm is not the highest, the total delay of the system is significantly reduced. In the future work, we will continue to explore new ways to achieve more flexible allocation of multi-dimensional resources.

\section{REFERENCES}

[1] N. H. Mahmood, M. Lauridsen, G. Berardinelli, D. Catania, and P. Mogensen, "Radio Resource Management Techniques for eMBB and mMTC Services in 5G Dense Small Cell Scenarios," in Proc. 2016 IEEE 84th Vehicular Technology Conference (VTC-Fall), Montreal, Sep. 2016, pp. 1-5.

[2] Thomas Jacobsen, Renato Abreu, Gilberto Berardinelli, Klaus Pedersen, and Preben Mogensen, "System Level Analysis of Uplink Grant-Free Transmission for URLLC," in Proc. 2017 IEEE Globecom Workshops (GC Wkshps), Singapore, Dec. 2017, pp. 1-6.

[3] D. Evans, "The internet of things: How the Next Evolution of the Internet is Changing Everything," CISCO white paper, 2011.
[4] P. K. Sinha and A. Dutta, "Multi-Satellite Task Allocation Algorithm for Earth Observation," in Proc. 2016 IEEE Region 10 Conference (TENCON), Singapore, Nov. 2016, pp. 403-408.

[5] P. Enge, "Satellite Navigation: Present and Future," URSI Radio Science Bulletin, vol. 2012, no. 341, pp. 5-9, Jun. 2012.

[6] V. A. Siris, Y. Thomas, and G. C. Polyzos, "Supporting the IoT over Integrated Satellite-Terrestrial Networks Using Information-Centric Networking," in Proc. 2016 8th IFIP International Conference on New Technologies, Mobility and Security (NTMS), Larnaca, Nov. 2016, pp. 1-5.

[7] Z. Qu, G. Zhang, H. Cao, and J. Xie, "LEO Satellite Constellation for Internet of Things," in IEEE Access, vol. 5, pp. 18391-18401, Sep. 2017.

[8] M. De Sanctis, E. Cianca, G. Araniti, I. Bisio, and R. Prasad, "Satellite Communications Supporting Internet of Remote Things," in IEEE Internet of Things Journal, vol. 3, no. 1, pp. 113-123, Feb. 2016.

[9] O. Kodheli, S. Andrenacci, N. Maturo, S. Chatzinotas, and F. Zimmer, "Resource Allocation Approach for Differential Doppler Reduction in NB-IoT over LEO Satellite," in Proc. 2018 9th Advanced Satellite Multimedia Systems Conference and the 15th Signal Processing for Space Communications Workshop (ASMS/SPSC), Berlin, Nov. 2018, pp. 1-8.

[10] R. Soua, M. R. Palattella, and T. Engel, "IoT Application Protocols Optimisation for Future Integrated M2M-Satellite Networks," in Proc. 2018 Global Information Infrastructure and Networking Symposium (GIIS), Thessaloniki, Greece, Oct. 2018, pp. 1-5.

[11] W. Chien, C. Lai, M. S. Hossain, and G. Muhammad, "Heterogeneous Space and Terrestrial Integrated Networks for IoT: Architecture and Challenges," in IEEE Network, vol. 33, no. 1, pp. 15-21, Jan. 2019.

[12] H. Zhang, Y. Xiao, S. Bu, D. Niyato, F. R. Yu, and Z. Han, "Computing Resource Allocation in Three-Tier IoT Fog Networks: A Joint Optimization Approach Combining Stackelberg Game and Matching," in IEEE Internet of Things Journal, vol. 4, no. 5, pp. 1204-1215, Oct. 2017.

[13] H. Zhang, Y. Zhang, Y. Gu, D. Niyato, and Z. Han, "A Hierarchical Game Framework for Resource Management in Fog Computing," in IEEE Communications Magazine, vol. 55, no. 8, pp. 52-57, Aug. 2017.

[14] J. Zhang, X. Zhang, M. A. Imran, B. Evans, Y. Zhang, and W. Wang, "Energy Efficient Hybrid Satellite Terrestrial 5G Networks with Software Defined Features," in Journal of Communications and Networks, vol. 19, no. 2, pp. 147-161, Apr. 2017.

[15] Y. Shi, Y. Cao, J. Liu and N. Kato, "A Cross-Domain SDN Architecture for Multi-Layered Space-Terrestrial Integrated Networks," in IEEE Network, vol. 33, no. 1, pp. 29-35, Jan. 2019.

[16] Bohao Feng, Huachun Zhou, Hongke Zhang, Guanwen Li, Haifeng Li, Shui Yu, and Han-Chieh Chao, "HetNet: A Flexible Architecture for Heterogeneous Satellite-Terrestrial Networks," in IEEE Network, vol. 31, no. 6, pp. 86-92, Nov. 2017.

[17] J. Liu, Y. Shi, Z. Md. F., and N. Kato, "Space-Air-Ground Integrated Network: A Survey", in IEEE Communications Surveys and Tutorials, vol. 20, no. 4, pp. 2714-2741, Oct. 2018

[18] A. Narula-Tam, H. Yao, J. Pandya, and J. Wysocarski, "Resource Request Strategies for Dynamic Resource Allocation in a Satellite Network," in Proc. MILCOM 2007 - IEEE Military Communications Conference, Orlando, Oct. 2007, pp. 1-7.

[19] L. Boero, M. Marchese, and F. Patrone, "The Impact of Delay in Software-defined Integrated Terrestrial-satellite Networks," in China Communications, vol. 15, no. 8, pp. 11-21, Aug. 2018.

[20] X. Zhu, C. Jiang, W. Feng, L. Kuang, Z. Han, and J. Lu, "Resource Allocation in Spectrum-sharing Cloud Based Integrated TerrestrialSatellite Network," in Proc. 2017 13th International Wireless Communications and Mobile Computing Conference (IWCMC), Valencia, Jun. 2017, pp. 334-339.

[21] J. Liu, Y. Shi, L. Zhao, Y. Cao, W. Sun and N. Kato, "Joint Placement of Controllers and Gateways in SDN-Enabled 5GSatellite Integrated Network," in IEEE Journal on Selected Areas in Communications, vol. 36, no. 2, pp. 221-232, Feb. 2018.

[22] T. Li, H. Zhou, H. Luo, and S. Yu, "SERvICE: A Software Defined Framework for Integrated Space-Terrestrial Satellite Communication," in IEEE Transactions on Mobile Computing, vol. 17, no. 3, pp. 703-716, Mar. 2018. 
[23] Y. Cao, H. Guo, J. Liu, and N. Kato, ąroptimal Satellite Gateway Placement in Space-Ground Integrated Networksąs, in IEEE Network, vol. 32, no. 5, pp. 32-37, Sep. 2018.

[24] Y. Cao, Y. Shi, J. Liu, and N. Kato, "Optimal Satellite Gateway Placement in Space-Ground Integrated Network for Latency Minimization with Reliability Guarantee," in IEEE Wireless Cоттиnications Letters, vol. 7, no. 2, pp. 174-177, Apr. 2018.

[25] N. Kato, Z. Md. Fadlullah, F. Tang, B. Mao, S. Tani, A. Okamura, and J. Liu, "Optimizing Space-Air-Ground Integrated Networks by Artificial Intelligence", in IEEE Wireless Communications, vol. 26, no. 4, pp. 140-147, 2019.

[26] A. Kak, E. Guven, U. E. Ergin, and I. F. Akyildiz, "Performance Evaluation of SDN-Based Internet of Space Things," in Proc. 2018 IEEE Globecom Workshops (GC Wkshps), Abu Dhabi, Dec. 2018, pp. 1-6.

[27] K. N. Bui and J. J. Jung, "Cooperative Game Theoretic Approach for Distributed Resource Allocation in Heterogeneous Network," in Proc. 2017 International Conference on Intelligent Environments (IE), Seoul, Aug. 2017, pp. 168-171

[28] P. Semasinghe, S. Maghsudi, and E. Hossain, "Game Theoretic Mechanisms for Resource Management in Massive Wireless IoT Systems," in IEEE Communications Magazine, vol. 55, no. 2, pp. 121-127, Feb. 2017.

[29] D. K. Petraki, M. P. Anastasopoulos, H. Chen, and P. G. Cottis, "Distributed Resource Allocation for Delay-Sensitive Services in Satellite Networks Using Game Theory," in IEEE Transactions on Computational Intelligence and AI in Games, vol. 1, no. 2, pp. 134-144, Jun. 2009.

[30] X. He, H. Dai, P. Ning, and R. Dutta, "A multi-player Markov stopping game for delay-tolerant and opportunistic resource sharing networks," in Proc. The 35th Annual IEEE International Conference on Computer Communications, San Francisco, Apr. 2016, pp. $1-9$.

[31] G. Oddi, M. Panfili, A. Pietrabissa, L. Zuccaro, and V. Suraci, "A Resource Allocation Algorithm of Multi-cloud Resources Based on Markov Decision Process," in Proc. 2013 IEEE 5th International Conference on Cloud Computing Technology and Science, Bristol, Dec. 2013, pp. 130-135.

[32] S. M. Ahsan Kazmi, Nguyen H. Tran, Walid Saad, Zhu Han, Tai Manh Ho, Thant Zin Oo, and Choong Seon Hong, "Mode Selection and Resource Allocation in Device-to-Device Communications: A Matching Game Approach," in IEEE Transactions on Mobile Computing, vol. 16, no. 11, pp. 3126-3141, Nov. 2017.

[33] M. Chen, S. C. Liew, Z. Shao, and C. Kai, "Markov Approximation for Combinatorial Network Optimization," in IEEE Transactions on Information Theory, vol. 59, no. 10, pp. 6301-6327, Oct. 2013.

[34] S. Zhang, Z. Shao, M. Chen, and L. Jiang, "Optimal Distributed P2P Streaming Under Node Degree Bounds," in IEEE/ACM Transactions on Networking, vol. 22, no. 3, pp. 717-730, Jun. 2014

[35] H. W. Khun, "The Hungarian method for the assignment problem," in Naval Res. Logistics Quart, vol. 2, pp. 83íC97, Mar. 1955.

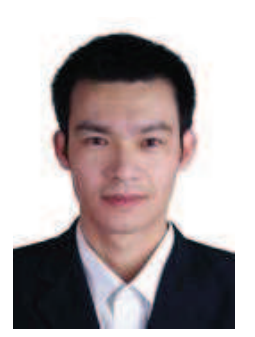

CHUNGANG YANG (S'09-M'11-SM'19) is a full professor at Xidian University, where he leads the research team of "GUIDE, Game, Utility, artificial Intelligent Design for Emerging communications". He received his Bachelor and Doctoral Degree in Xidian University, Xián, China, in 2006 and 2011, respectively. Between Sep. 2010 and March 2011, he held the visiting scholar position in the department of electrical and computer engineering at Michigan Technological University (MTU). Between March 2015 and March 2016, he held the visiting scholar position in the department of electrical and computer engineering at University of Houston (UH). Dr. Yang's research interests include resource and interference management and network optimization for cognitive radio networks, heterogeneous green cellular networks, with focus on game theory for wireless communication and computing networks. He has edited two books, and published 20+ journals and 20+ conferences. Dr. Yang's recent research interests are artificial intelligent B5G/6G wireless mobile networks, intent-driven/based networks (IDN/IBN), and spaceterrestrial networks (STN), and so on.

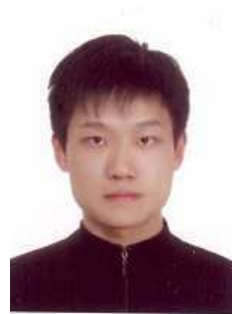

PENG GONG received the BS degree in Mechetronical Engineering from Beijing Institute of Technology, Beijing, China, in 2004, and the MS and Ph.D. degrees from the Inha University, Korea, in 2006 and 2010, respectively.

In July 2010, he joined the School of Mechetronical Engineering, Beijing Institute of Technology, China. His research interests include link/system level performance evaluation and radio resource management in wireless systems, information security, and the next generation wireless systems such as $5 \mathrm{G}, \mathrm{UWB}, \mathrm{MIMO}$, Cognitive radio and so on.

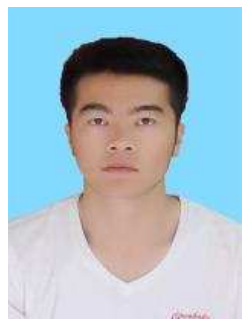

LIZHONG CHANG received the Bachelor's degree in Electronic Information Engineering from Guizhou University, Guizhou, China, in 2018 , where he is currently pursuing the master's degree in Information and Communication Engineering. He is also with the GUIDE Research Team, under the guidance of Dr. C. Yang. His research interests include wireless adhoc network and communication protocol.

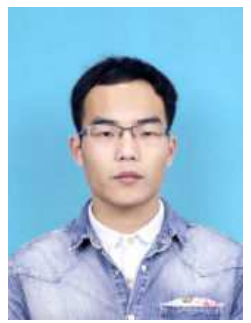

JUNQI SHAO received the Bachelor's degree in network engineering from North China University of Water Resources and Electric Power, Zhengzhou, China, in 2018, where he is currently pursuing the masterarís degree in information and communication engineering. $\mathrm{He}$ is also with the GUIDE Research Team, under the guidance of Dr. C. Yang. His research interests include wireless adhoc network and communication protocol. 


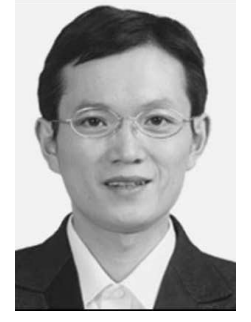

QIANG NI (M'04-SM'08) received his Ph.D. degree in Engineering from Huazhong University of Science and Technology, Wuhan, in 1999. He is currently a Full Professor and the Head of Communication Systems Research Group at the School of Computing and Communications, Lancaster University, Lancaster, UK. His research interests include future generation communications and networking systems, energy and spectrum efficient green wireless communications, 5G, SDN, game theory, heterogeneous networks, cognitive radio network systems, ultra-dense networks, cloud networks, energy harvesting, IoTs, vehicular networks, big data analytics, etc. He had published more than 180 research papers in international journals and conferences. He was an IEEE 802.11 Wireless Standard Working Group Voting Member and a contributor to various IEEE Wireless Standards.

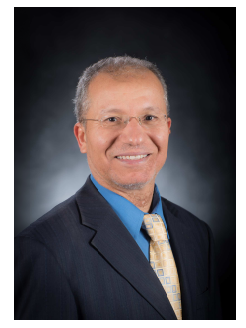

MOHSEN GUIZANI (S'85-M'89-SM'99F'09) received the B.S. (with distinction) and M.S. degrees in electrical engineering, the M.S. and $\mathrm{Ph} . \mathrm{D}$. degrees in computer engineering from Syracuse University, Syracuse, NY, USA, in 1984, 1986, 1987, and 1990, respectively. He is currently a Professor and the ECE Department Chair at the University of Idaho, USA. Previously, he served as the Associate Vice President of Graduate Studies, Qatar University, Chair of the Computer Science Department, Western Michigan University, and Chair of the Computer Science Department, University of West Florida. He also served in academic positions at the University of Missouri-Kansas City, University of Colorado- Boulder, Syracuse University, and Kuwait University. His research interests include wireless communications and mobile computing, computer networks, mobile cloud computing, security, and smart grid. He currently the Editorin- Chief of the IEEE Network, serves on the editorial boards of several international technical journals and the Founder and the Editor-in-Chief of Wireless Communications and Mobile Computing journal (Wiley). He is the author of nine books and more than 500 publications in refereed journals and conferences. He guest edited a number of special issues in IEEE journals and magazines. $\mathrm{He}$ also served as a member, Chair, and General Chair of a number of international conferences. He received the teaching award multiple times from different institutions as well as the best Research Award from three institutions. He received the 2017 IEEE ComSoc Recognition Award for his contribution to Wireless Communications. He was the Chair of the IEEE Communications Society Wireless Technical Committee and the Chair of the TAOS Technical Committee. He served as the IEEE Computer Society Distinguished Speaker from 2003 to 2005. He is a Fellow of IEEE and a Senior Member of ACM.

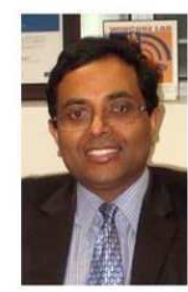

Alagan Anpalagan received the B.A.Sc. M.A.Sc. and Ph.D. degrees in Electrical Engineering from the University of Toronto, Canada. He joined the Department of Electrical and Computer Engineering at Ryerson University in 2001 and was promoted to Full Professor in 2010. He served the department as Graduate Program Director (2004-09) and the Interim Electrical Engineering Program Director (2009-10). During his sabbatical (2010-11), he was a Visiting Professor at Asian Institute of Technology and Visiting Researcher at Kyoto University. Dr. Anpalagan's industrial experience includes working at Bell Mobility, Nortel Networks and IBM Canada. Dr. Anpalagan directs a research group working on radio resource management (RRM) and radio access \& networking (RAN) areas within the WINCORE Lab. His current research interests include cognitive radio resource allocation and management, wireless cross layer design and optimization, cooperative communication, M2M communication, small cell networks, energy harvesting and green communications technologies. He is a Senior Member of the IEEE. 